\title{
La lutte contre l'immigration irregulière. Quelques reprises sur l'expérience française
}

\author{
François Dieu
}

\section{Riassunto}

L'immigrazione illegale è oggetto, in Francia, di molte polemiche. Quest'articolo propone alcuni spunti di riflessione sull'attività di contrasto nei confronti di questa particolare forma di criminalità i cui autori, anche se spesso vittime di reti criminali, sono l'oggetto di un processo di stigmatizzazione che giustifica appositi interventi delle forze di polizia.

\section{Résumé}

L'immigration irrégulière fait l'objet, en France, de nombreuses controverses. Cet article apporte quelques repères sur l'action répressive menée contre cette forme particulière de délinquance, dont les auteurs, bien que souvent victimes de filières criminelles, font l'objet d'une stigmatisation justifiant le déploiement d'un dispositif policier.

\section{Abstract}

The illegal immigration is the source of much controversy in France. This article provides an overview of the repressive action against this particular form of criminality, whose authors, although often victims of criminal networks, are the subjects of a stigmatization process justifying the deployment of police measures.

Key words: illegal immigration; repressive action; criminal networks; victims; France.

La France est une terre d'accueil et un pays d'immigration. Elle a délivré, en 2012, pas moins de 200000 titres de séjours. Elle compte actuellement 5,5 millions d'immigrés, soit 8,6\% de sa population, mais plus d'un Français sur quatre est « issu de l'immigration » (immigré ou avec au moins un parent immigré). C'est le cas de l'ancien président de la République (Nicolas Sarkozy), et actuellement, du premier ministre (Manuel Valls) ou encore de la ministre de l'Education nationale (Najat Vallaud-Belkacem). L'immigration est un phénomène social complexe et passionnel, en lien avec des problématiques sociétales fondamentales que sont la construction européenne et l'histoire coloniale, les politiques d'intégration et les contours de la nationalité et de la citoyenneté. Il ne s'agit pas ici, bien évidemment, d'évoquer toutes ces questions en lien avec les politiques d'immigration et le statut de l'immigré, mais seulement d'apporter quelques repères sur le volet répressif des politiques menées en ce domaine, c'est-à-dire la lutte contre l'immigration irrégulière, appréhendée comme une action répressive à la fois controversée (1) et malaisée (2).

\section{Une action répressive controversée.}

Le gouvernement français a mis fin, au début des années 70, aux régularisations automatiques des travailleurs étrangers (un étranger en situation irrégulière voyait jusque-là sa situation relativement vite régularisée par une administration bienveillante envers les besoins

\footnotetext{
- Professeur des universités, Centre d'Études et de Recherches sur la Police, Université Toulouse 1 Capitole, France.
} 
de main d'œuvre) et a instauré progressivement une politique dite de «maitrise des flux migratoires ». Derrière cette expression bureaucratique se profile la volonté de limiter le nombre de nouveaux immigrés (immigration économique, familiale et étudiante), ce qui est particulièrement manifeste dans un contexte de crise économique et de chômage endémique, avec comme corollaire la « chasse » aux immigrés en situation irrégulière, par une plus grande vigilance à l'entrée sur le territoire (avec des contrôles à la sortie d'avion dans les aéroports, notamment Roissy/CDG ou dans les trains entre la France et l'Italie et l'Espagne), mais aussi par des mesures de contrôle visant ceux déjà installés (l'arrestation des immigrés en situation irrégulière pouvant alors intervenir à la faveur des contrôles d'identité avec la problématique des «contrôle au faciès", de la constatation d'infractions commises, voire au guichet des préfectures).

A l'appui de cette politique répressive, l'immigration donne lieu à un encadrement normatif (c'est le cas aujourd'hui avec la loi du 11 mai 1998 relative à l'entrée et au séjour des étrangers en France et au droit d'asile et le Code de l'entrée et du séjour des étrangers et du droit d'asile), avec, d'un côté, un volet légal (délivrance de visas et de titres de séjour, regroupement familial, accueil des demandeurs d'asile, acquisition de la nationalité), de l'autre, un volet déviant, une situation d'immigration irrégulière pouvant résulter de deux cas de figure: soit un étranger est entré de manière clandestine sur le territoire avec l'aide ou non de tiers ou d'une organisation criminelle, soit il est demeuré après l'expiration de son titre de séjour (avec éventuellement un refus de la préfecture de renouveler ce titre). La grande majorité des étrangers en situation irrégulière relève de cette seconde catégorie, ce qui démontre la fausseté de l'idée d'une intrusion massive par des circuits d'immigration parallèle. L'étranger en situation irrégulière est souvent une personne entrée légalement en France, directement ou par un pays de l'espace Schengen. A cet égard, lorsqu'un Etat européen fait entrer sur son sol un étranger sur la base de documents qui peuvent avoir été falsifiés, il est responsable de la délivrance d'une autorisation de séjour valable pour les 26 Etats qui ont supprimé leurs frontières et leurs contrôles.

A partir du moment où il décide de poursuivre illégalement son séjour en France, il devient alors un «clandestin» ou un "sans papier», privé notamment du droit au travail. Cette expression a connu une large diffusion auprès du grand public à la suite de l'occupation, dans les années 1990, d'églises parisiennes par des collectifs («Des papiers pour tous!», etc.) revendiquant une régularisation massive de leur situation (Saint-Ambroise le 18 mars 1996 et Saint-Bernard du 28 juin au 23 août 1996).

En dépit de divergences entre la gauche et la droite sur la question de l'immigration, la première étant plus favorable à des régularisations massives alors que la seconde prend plutôt position sur un durcissement des conditions d'accès et de séjour et sur la mise en œuvre de mesures d'expulsions plus systématiques, il existe un consensus des gouvernements successifs sur la nécessité de lutter contre l'immigration régulière, ce qu'a exprimé Manuel Valls, alors ministre de l'Intérieur, dans sa circulaire aux préfets du 11 mars 2013: «La lutte contre l'immigration 
irrégulière est une priorité. Elle doit être menée avec efficacité et détermination ».

En matière d'immigration irrégulière, la France est à la fois une zone de transit, principalement pour les ressortissants des pays de la Corne de l'Afrique (Somalie, Soudan, Erythrée, Ethiopie) et du Moyen Orient (Iran, Irak, Syrie) en route vers le Royaume-Uni et les pays scandinaves, mais aussi une zone d'installation durable, principalement pour les ressortissants des pays du Maghreb (Tunisie notamment depuis 2010).

Le migrant est généralement considéré comme un délinquant mais aussi comme une victime, un statut ambigu induit par la Convention des Nations Unies contre la criminalité transnationale organisée (convention de Palerme, 12-15 décembre 2000). En effet, au-delà de sa situation personnelle et des raisons l'ayant conduit à fuir son pays d'origine (misère, persécutions politiques, ethniques et religieuses, instabilité chronique, etc.), ce qui plaide pour une approche généreuse et humaniste, il peut être aussi la victime de deux types de criminalité, à savoir, d'une part, le trafic illégal des migrants (avec des passeurs peu scrupuleux exploitant leur situation pour leur soutirer de fortes sommes, voire les maltraitant et les abandonnant sans vivres en pleine mer sur des embarcations de fortune, voire en coulant ces dernières et en les condamnant ainsi à une mort certaine), d'autre part, le trafic des êtres humains (avec des réseaux susceptibles d'exploiter des migrants en les faisant travailler illégalement ou encore en les contraignant à se livrer à des activités criminelles, de trafic de stupéfiants, de mendicité ou de prostitution). Les conditions de vie des étrangers en situation irrégulière demeurent difficiles même lorsqu'ils sont parvenus à pénétrer sur le territoire national. En matière d'hébergement, ils subissent pleinement les escroqueries des «marchands de sommeil». Comme le notait la Cour des comptes en 2004 : «Privés de certains droits essentiels et en particulier de celui de travailler régulièrement, les étrangers en situation irrégulière sont exposés, selon les cas, à l'exploitation par des employeurs clandestins dépourvus de scrupules, à l'obligation de s'adonner à des activités lucratives mais illégales (souvent délictuelles, parfois criminelles), ou à la plus désespérante précarité». Lorsqu'ils sont victimes d'infraction, ils ne peuvent pas, bien évidemment, accéder à la police et à la justice, ce qui fait d'eux la proie de toutes les formes de délinquance, avec la certitude d'une certaine impunité.

La lutte contre l'immigration irrégulière est porteuse de cette contradiction, avec, d'un côté, des mesures de contrôle et d'interpellation (avec retenue et internement) et des poursuites judiciaires, mais aussi l'exigence d'une nécessaire protection du migrant en dépit de sa responsabilité propre (et d'une relation qui peut être ambiguë, voire une complicité avec les passeurs), de telle sorte que ce qui est mis en avant par les instances en charge de cette politique est la lutte contre les filières d'immigration illégale, considérée comme une activité criminelle (en 2013, 201 filières ont été démantelées, en France, par les services de police). Le titre d'un rapport sénatorial de 2006 portant sur ces questions est assez révélateur de cette approche qui oscille entre répression et humanisme: «Immigration clandestine: une réalité inacceptable, une réponse ferme, juste et humaine ». 
Thème privilégié de l'extrême droite qui, depuis des lustres, a fait de l'immigration son fonds de commerce (avec une assimilation fallacieuse entre immigration et immigration irrégulière, la seconde étant présentée comme la conséquence inévitable de la première), une immigration jugée responsable, pêle-mêle, de la recrudescence du chômage, des déficits publics, de la délinquance, des communautarismes, et surtout d'un recul identitaire irréversible, il donne lieu en permanence à des controverses et des débats, entretenus par des épisodes souvent tragiques, révélateurs des difficultés, mais aussi des ambiguités de cette politique publique répressive pas forcément assumée. Pour ne prendre que trois épisodes les plus médiatisés.

- Le «camp de réfugiés » de Sangatte : En 1999, en accord avec les autorités préfectorales, est créé à Sangatte, station balnéaire du Pas de Calais, un centre d'hébergement et d'accueil d'urgence humanitaire administré par la CroixRouge. Ce centre, connu sous le nom de « centre de Sangatte » est installé dans une ancienne usine d'Eurotunnel et possède un équipement limité prévu pour accueillir 200 personnes. Or, les besoins de la population de migrants (principalement Afghans, Iraniens, Irakiens, Kurdes et Kosovars) sont tels qu'on a compté jusqu'à 1600 personnes hébergées dans des conditions précaires, sources de tensions, notamment avec la population locale. En novembre 2002, le ministre de l'Intérieur, Nicolas Sarkozy, sous la pression britannique, ordonne sa fermeture et son démantèlement, mais le problème reste entier pour le Calaisis : de nouveaux immigrés illégaux, qui sont entrés dans l'espace Schengen principalement par la Grèce qui subit une forte pression migratoire à sa frontière avec la Turquie, continuent d'affluer malgré la fermeture définitive du centre le 16 décembre 2002. Les migrants (environ 600) sont depuis lors dispersés à divers endroits du littoral dans des abris de fortune. Si la surveillance du port de Calais et des moyens de passage outreManche est devenue de plus en plus rigoureuse, elle se heurte à des impératifs économiques, des contrôles trop minutieux pouvant ralentir le trafic particulièrement dense (bateaux, Shuttle, navettes du tunnel) sur et sous la Manche. La rigueur des contrôles est donc toute relative et les étrangers qui cherchent à passer en Angleterre finissent, pour une bonne part, à y parvenir. En revanche, le passage n'est pas immédiat: il leur faut persévérer, en moyenne, trois à quatre semaines actuellement, à raison de tentatives qui peuvent être quotidiennes. Les candidats au passage outre-Manche, entrés en France par la Belgique ou l'Italie, sont donc, après leur long périple, bloqués dans ce véritable « sas », à quelques encablures de la terre promise britannique, objet d'une attention médiatique soutenue et de fortes crispations avec la population locale.

- L' «échouage» de l'East Sea : le 17 février 2001, un vieux vraquier rouillé, battant pavillon cambodgien, s'échoue près de Saint-Raphaël. Dans ses cales, plus de 900 réfugiés kurdes, dont la moitié d'enfants, ayant voyagé huit jours, avant d'être abandonnés par leurs passeurs. Les «boat people» kurdes affirment qu'ils ont fui l'Irak de Saddam Hussein. L'échouage de l'East Sea est le premier débarquement sauvage de clandestins sur les côtes françaises. Les réfugiés sont regroupés dans une caserne à Fréjus. Confrontées pour la première fois à l'arrivée massive d'étrangers en situation irrégulière sur le 
territoire, les autorités françaises sont placées face à un véritable imbroglio administratif et juridique. Dans un premier temps, le ministère de l'Intérieur choisit de suivre la procédure d'admission au titre de l'asile sollicitée par les Kurdes. Munis d'un sauf-conduit de huit jours, ils tentent alors de quitter la France clandestinement. Parmi eux, en effet, beaucoup veulent se rendre en Allemagne où réside une importante communauté kurde, mais aussi en Suisse ou encore aux Pays-Bas. Le 20 avril, soit deux mois après le naufrage de l'East Sea, le quotidien «La Croix» devait révéler que les Kurdes n'étaient pas irakiens, comme ils l'avaient d'abord affirmé, mais syriens.

- La «rafle de Leonarda» : une affaire politique au fort retentissement médiatique s'est développée à la suite de l'expulsion vers le Kosovo, le 9 octobre 2013, d'une famille immigrée rom dont une fille mineure, Leonarda Dibrani, a été interpellée lors d'une sortie scolaire. Décrite par certains médias comme s'étant déroulée sous les yeux de ses camarades de classe, l'interpellation de cette collégienne qui vivait en France depuis près de cinq ans provoque une vague d'indignations et de manifestations à travers le pays. À quelques mois de scrutins municipaux et européens, l'affaire a pris les dimensions d'une crise politique après une communication maladroite du président de la République qui déclarait, le 19 octobre, autoriser la jeune fille à poursuivre ses études en France, mais sans sa famille. Le ministre de l'Intérieur annonçait le 23 octobre 2013 qu'il envisageait une réforme du droit d'asile en France, tandis que la porte-parole du gouvernement déclarait que cette affaire était résolue. En mai 2014, la presse a fait état de l'intention de Leonarda de revenir en France sous une nouvelle identité et avec une nouvelle nationalité (croate).

\section{Une action répressive malaisée.}

Objet de controverses, l'immigration irrégulière demeure un phénomène encore méconnu, notamment au plan de son ampleur exacte, ce qui autorise la prolifération des prises de position les plus irrationnels et dogmatiques. Il est, en effet, pratiquement impossible de quantifier même approximativement le nombre de personnes étrangères entrées ou séjournant de façon irrégulière, ce nombre étant estimé entre 200000 et 400000 . Il ne s'agit que d'estimations car, par la nature même de cette immigration, les chiffres sont difficiles à évaluer. On ne dispose que d'indicateurs généralement en lien avec l'activité des services répressifs, et donc qui sont surtout révélateurs non de l'ampleur du phénomène, mais des moyens mobilisés pour $\mathrm{y}$ faire face et des résultats éventuellement obtenus :

- les refoulements à la frontière : 11508 en 2012 (17 628 en 2008), principalement des Algériens, Brésiliens (Guyane), Marocains et Tunisiens ;

- les mesures d'éloignement: 21847 retours contraints en 2012, 4954 retours contraints aidés et 10021 départs volontaires aidés (pour l'aide au retour volontaire, 4859 bénéficiaires en 2012, le montant de l'aide est de 2000 euros pour un adulte seul, 3500 euros pour un couple, 1000 euros par enfant mineur jusqu'au troisième inclus et 500 euros à partir du quatrième enfant). Les ressortissants roumains représentent la nationalité la plus éloignée (30\%), cet éloignement intervenant pour des motifs de troubles à l'ordre public, d'infractions à la 
législation sur le travail ou à la suite de la perte du droit de séjour au-delà des trois mois de présence sur le territoire national. Les ressortissants originaires du Maghreb constituent l'autre catégorie la plus importante (24\%). En l'absence de passeport, les reconduites à la frontière nécessitent la délivrance de laissezpasser consulaires par l'Etat d'origine de l'étranger, ce qui n'est pas chose aisée : en 2012, 6515 demandes pour 2403 laisser-passer obtenus dans les délais utiles (soit 37\%), les pays les plus coopératifs étant l'Afghanistan (71\%), l'Angola (56\%) ou encore la Chine (53\%) et les moins coopératifs, la Côte d'Ivoire $(0 \%)$, le Gabon (3,5\%) ou encore l'Irak (12\%) ;

- les personnes mises en cause pour faits de délinquance : deux délits sont prévus par le Code pénal et comptabilisés par un état statistique policier (Etat « $4001 »)$ qui comporte 107 index : index 70 (aide à l'entrée irrégulière, à la circulation et au séjour irrégulier des étrangers) : 6064 en 2012 ; index 69 (délits d'entrée et de séjour des étrangers) : 69453 en 2012 (-20\% par rapport à 2010) ;

- les placements en centres de rétention administrative $(\mathrm{CRA})^{1}$ : étrangers en situation

\footnotetext{
1 Les centres de rétention administrative (CRA) ont été créés par la loi du 29 octobre 1981. Il existe actuellement 25 centres de rétention administrative sur l'ensemble du territoire français, qui disposent de 1755 places dont 184 outre-mer. Le CRA est un bâtiment surveillé par la police dans lequel l'administration (le préfet) peut retenir, pour une durée limitée, et sous contrôle juridictionnel, les étrangers faisant l'objet d'une procédure d'éloignement et ne pouvant pas quitter immédiatement le territoire. Cette phase de placement est appelée la "rétention administrative ». Le CRA ne relève pas de l'administration pénitentiaire dans la mesure où la privation de liberté ne résulte pas d'une décision judiciaire mais administrative. Une personne peut rester en rétention au maximum quarante-cinq jours ; au-delà de cette durée, l'étranger que l'administration n'a pas reconduit doit être remis
}

irrégulière en attente de reconduite à la frontière sous le coup d'une obligation de quitter le territoire, d'un arrêté de reconduite à la frontière, d'un arrêté d'expulsion préfectoral ou ministériel, d'une mesure de réadmission ou condamnés à une peine d'interdiction du territoire : 39989 en 2012, dont 4088 mineurs ($33 \%$ par rapport à 2010) ;

- les arrêtés préfectoraux de reconduite à la frontière et obligations de quitter le territoire: 82806 en 2012 dont 19290 exécutés (26\%) (+15\% par rapport à 2010).

Aussi peut-on considérer avec plus d'attention, le nombre de bénéficiaires de l'aide médicale d'Etat (prise en charge depuis 2000 des frais médicaux des étrangers en situation irrégulière), qui semble plus proche du nombre effectif

en liberté. Les étrangers présents dans les CRA peuvent, dans certaines conditions, exercer des recours devant la justice pénale ou administrative pour contester leur placement en rétention ou leur refus de séjour. Pour exercer leurs droits, ils peuvent être assistés par une association présente dans le CRA (CIMADE) et un avocat. En France, la durée moyenne de rétention est de 11 jours $(2$ mois en Espagne, au Portugal et en Italie, 1 an mois en Grèce, 8 mois en Belgique, 6 mois en Autriche et 18 mois en Allemagne ; la Finlande, les Pays-Bas et la GrandeBretagne ne connaissant aucune limitation de durée). Dans une décision du 3 septembre 1986, le Conseil constitutionnel a rappelé que le placement en rétention, s'il est justifié, doit respecter les droits de la défense, être limité dans le temps et être lié à l'impossibilité de procéder immédiatement à l'exécution de la mesure d'éloignement. Les CRA sont placés sous le contrôle du Contrôleur général des lieux de privation de liberté, institué par la loi du 30 octobre 2007. Cette autorité administrative indépendante est chargée de « contrôler les conditions de prise en charge et de transfèrement des personnes privées de liberté, afin de s'assurer du respect de leurs droits fondamentaux ». Elle exerce sa mission au moyen de visites programmées ou inopinées des lieux dans lesquelles ces personnes sont détenues, retenues, maintenues ou placées. Le Procureur de la République, le juge des libertés et de la détention, les parlementaires disposent également d'un droit de visite. 
d'immigrés en situation irrégulière, soit 252437 bénéficiaires en 2012, pour un coût d'environ 587,5 millions d'euros (+10\% depuis 2010).

La priorité de la répression réside, au moins officiellement, dans la lutte contre les filières. La loi française prévoit que le fait de séjourner de manière irrégulière sur le territoire constitue un délit passible d'un an de prison, de $3750 €$ d'amende et de 3 ans d'interdiction du territoire. Mais la jurisprudence a déduit en 2012 de la jurisprudence de la Cour de Justice de l'Union Européenne que cette infraction ne peut être punie d'une peine d'emprisonnement parce qu'une telle sanction va à l'encontre des objectifs de la directive retour. S'agissant de la lutte contre les filières, elle pâtit de l'absence de définition juridique de la «filière », les seules qualifications pénales disponibles étant celles de la «bande organisée » ou de l' " association de malfaiteurs ». Par ailleurs, les peines encourues s'avèrent relativement modestes :

- l'aide à l'entrée, à la circulation et au séjour irréguliers d'étrangers en France: 5 ans d'emprisonnement et de 30000 euros d'amende; lorsqu'elle est commisse en bande organisée : 10 ans d'emprisonnement et 750000 euros d'amende ;

- le faux et usage de faux documents administratifs : 5 ans d'emprisonnement et 75000 euros d'amende ;

- l'association de malfaiteurs dans le but de commettre l'infraction d'aide à l'immigration en bande organisé et l'emploi d'étrangers sans titre en bande organisée : 10 ans d'emprisonnement et 150000 euros d'amende.

L'activité des services de police a été amenée à intégrer, ces dernières années, les décisions de la
Cour de Justice de l'Union Européenne, notamment l'arrêt du 22 juin 2010 sur la limitation des contrôles d'identité dans la bande des vingt kilomètres d'une frontière et l'arrêt du 6 décembre 2011 (et arrêt de la Cour de Cassation du 5 juillet 2012) sur l'interdiction de mettre en garde à vue des étrangers en situation irrégulière, auxquels une mesure administrative invitant à quitter le territoire a été notifiée et pour lesquels aucune infraction connexe n'a été relevée (possibilité seulement d'une retenue de 16 heures pour vérification selon la loi du 31 décembre 2012).

Depuis le début des années 1990, un dispositif policier a été constitué pour lutter plus efficacement contre l'immigration irrégulière :

- 1992: déploiement d'une cinquantaine de brigades mobiles de recherche (BMR) de la Police de l'air et des frontières, entièrement dédiées à la lutte contre l'immigration irrégulière ;

- 1994: changement d'appellation de la PAF (Police de l'air et des frontières) en DICCILEC: Direction centrale du contrôle de l'immigration et de la lutte contre l'emploi des clandestins; redevenue en 1999 la PAF: Police aux frontières ;

- 1996 : mise en place de l'Office central pour la répression de l'immigration irrégulière et l'emploi d'étrangers sans titre (OCRIEST) rattaché à la Direction centrale de la police judiciaire/DCPJ, avec notamment un pôle opérationnel (qui s'articule en cinq groupes opérationnels à thématique géographique : Chine, Asie méridionale, Asie, MoyenOrient/Europe, Afrique/Amérique du Sud, liée à la nationalité d'origine des trafiquants de migrants) : animation de la lutte contre les 
filières d'immigration irrégulière, contre les réseaux structurés d'emploi d'étrangers sans titre de séjour et/ou d'autorisation de travail et contre les réseaux de fraude documentaire favorisant l'immigration irrégulière et le travail illégal; identification des menaces en termes de flux migratoires ;

- 2011: création de l'Unité de coordination opérationnelle de lutte contre le trafic et l'exploitation des migrants (UCOLTEM) rattaché à la Direction générale de la police nationale/DGPN : sur la modèle de l'UCLAT en matière de lutte antiterroriste, dirigée par le chef de l'OCRIEST, coordination de l'action de l'ensemble des services concernés: diplomatie (ministère des affaires étrangères et européennes); services de renseignements (Direction générale du renseignement extérieur/DGSE, Direction générale du renseignement intérieur/DGRI); services d'enquête (Direction de la coopération internationale/DCI, Direction centrale de la sécurité publique/DCSP, Direction centrale de la police judiciaire/DCPJ, Direction centrale de la police aux frontières/DCPAF, Direction générale de la gendarmerie nationale/DGGN, Douanes); avec trois missions principales: la centralisation, l'exploitation et la diffusion d'informations opérationnelles ; la coordination interservices (liaisons, recoupements de renseignements); la coopération internationale. Les autres services intervenant en ce domaine sont l'Office central pour la répression de la traite des êtres humains (OCRTEH) de la DCPJ pour les questions d'exploitation sexuelle et de prostitution, et l'Office central de lutte contre le travail illégal (OCLTI) de la DGGN pour les questions d' « esclavage domestique ».
Cette réponse policière s'inscrit également dans le cadre de la coopération européenne, en particulier sous la forme d'échanges de renseignements opérationnels au niveau d'EUROPOL et par la participation aux ateliers de travail et aux productions de FRONTEX.

La question de la sur-délinquance des immigrés fait débat dans la société française, mais aussi dans la communauté criminologique. La surreprésentation des immigrés et personnes issues de l'immigration dans les statistiques criminelles et la population carcérale résulte, en l'absence de statistiques fiables, de constats empiriques, avec comme explication, d'une part, la conséquence logique des situations d'exclusion sociale et de déficit d'intégration, d'autre part, la possible mobilisation plus importante à leur encontre des institutions policières et des juridictions répressives, de telle sorte que cette situation a pu être constatée au niveau des diverses vagues d'immigration qu'a connues la France depuis la fin du dix-neuvième siècle. S'agissant de l'immigration irrégulière, elle s'accompagne principalement de types d'infractions: le travail illégal et la fraude à l'identité, la répression de ces infractions étant d'ailleurs une voie plus détournée de la lutte contre l'immigration irrégulière.

En matière de travail illégal, la loi du 16 juin 2011 relative à l'immigration, à l'intégration et à la nationalité a introduit, à l'encontre des employeurs, des sanctions pénales (l'emploi d'étrangers sans titre est puni d'un emprisonnement de 5 ans et d'une amende de 15000 euros) et administratives (exclusion et remboursement des aides ou subventions ; fermeture temporaire d'établissements; exclusion des marchés publics), tout en 
prévoyant des mesures pour protéger les droits des étrangers sans titre (en termes notamment de recouvrement de leurs salaires et indemnités). En 2012, 13954 personnes ont été mises en cause pour emploi d'étrangers sans titre.

S'agissant de la fraude à l'identité et de la fraude documentaire, on assiste, ces dernières années, à une recrudescence importante des faits constatés : 14826 en 2009 à 21618 en 2012, principalement des contrefaçons (7 611) et des falsifications (8 424) de titres de séjour (1 264), de cartes d'identité (1 030) et de passeports (599), se traduisant par la mise en cause de 10907 personnes (dont 58\% d'étrangers). La fraude à l'identité ne concerne pas seulement la législation sur les étrangers, mais elle est aussi un moyen associé à la commission d'autres infractions (escroqueries, abus de confiance, fraudes aux prestations sociales, infractions à la législation du travail, etc.). Un dispositif de coopération a été développé au niveau européen (groupes «faux documents» et «fraude à l'identité» de l'Union européenne; agence FRONTEX ; Comité article 6 sur la sécurisation des documents de voyage : groupe e-MOBIDIG du Joint Research Center de la Commission européenne; groupe EDEWG du réseau ENFSI; base de données FADO) et international (accords bilatéraux; Immigration Fraud Conférence).

Bien qu'il soit surtout une victime exploitée par les réseaux criminels, qui commet incidemment plutôt une infraction «administrative » (en termes de droit d'entrée et de séjour) que " pénale », le «sans papier» n'en est pas moins appréhendé comme un personnage menaçant dans la société française d'aujourd'hui. Il dérange, inquiète et fait peur, alors même que sa présence ne constitue, en réalité, qu'un préjudice matériel assez limité, en termes notamment de travail illégal, d'assistance et de prise en charge des dépenses de santé. Parce qu'il est issu de pays en proie à la guerre et au terrorisme, ou plus prosaïquement à la misère et au sousdéveloppement, dans l'imaginaire collectif parasité par les discours les plus alarmistes, il porte en lui, à son corps défendant, les germes de l'importation de ces fléaux dans l'hexagone. Cette stigmatisation a conduit au renforcement de la répression pénale à l'encontre des immigrés irréguliers, figure criminelle toute désignée de la mondialisation et de l'ouverture des frontières.

Comme pour toutes les formes de délinquance, la société française se complet en la matière dans une confusion entre les «réponses» et les « solutions » : s'il est nécessaire de déployer des réponses policières et judiciaires à la question de l'immigration irrégulière, on ne peut imaginer, bien évidemment, qu'elles permettront de solutionner ce phénomène, contrairement aux actions qui peuvent être conduites, par exemple, en matière de développement solidaire, avec la mobilisation de moyens au profit du développement local dans les pays d'origine des immigrants clandestins. Par ailleurs, en ce domaine, comme d'ailleurs dans celui des réponses, la raison commande de ne pas se limiter des parades et ripostes nationales isolées, pour ne pas dire égoïstes, par la détermination et la mise en œuvre d'une approche globale sous la forme de politiques collectives, au moins dans le cadre européen. 


\section{Références.}

- AA.VV., "Politique de l'immigration», Regards sur l'Actualité, La documentation Française, n³26, décembre 2006.

- AA.VV., "Immigration et sécurité », Cabiers de la Sécurité, nº17-18, juillet-décembre 2011.

- AA.VV., Les étrangers en France. Année 2012, rapport établi en application de l'article L111.10 du Code de l'entrée et du séjour des étrangers et du droit d'asile.

- Bisi R., «Migrations et société globalisée », numéro spécial : «Immigration et sécurité », Cabiers de la Sécurité, n¹7-18, juilletdécembre 2011, pp. 32-42.

- Dubin L. (sous la dir. de), La Légalité de la lutte contre l'immigration irrégulière par l'Union Européenne, Bruylan, "Droit administratif», 2012.

- Fassin D., Morice A., Quiminal C. (sous la dir. de), Les Lois de l'inhospitalité. Les politiques de l'immigration à l'éprewve des sans-papiers, La Découverte, 1997.

- Fauvergue J-M, «La lutte contre le trafic de migrants en France», numéro spécial: "Immigration et sécurité", Cabiers de la Sécurité, nº17-18, juillet-décembre 2011, pp. 126-141.

- Siméant J., La Cause des sans-papiers, Presses de Sciences-Po, 1998.

- Noirel G., Le Creuset français. Histoire de l'immigration XIXe-XXe siècles, Seuil, «Points Histoire », 2006.

- Noiriel G., Réfugiés et sans-papiers: La République face au droit d'asile XIX'e-XXe siècle, Hachette, 2006.

- Sénat, Rapport de la Commission d'enquête, «Immigration clandestine: une réalité inacceptable, une réponse ferme, juste et humaine », Georges Othily et François-Noël Buffet, 2006.

- Tripier M., Réa A., Sociologie de l'immigration, La Découverte, Repères, 2008. 